\title{
New Concept of PQR-Coordinate on Power Transformation for Harmonics Reducing
}

\author{
Indriarto Yuniantoro ${ }^{1}$, Rudy Setiabudy ${ }^{1}$, and Ridwan Gunawan ${ }^{1}$
}

\begin{abstract}
Power transformation of pqr-coordinate modified by Euler angle rotation method used in analysis of the IGBT active filter circuits as the instantaneous power theory is reviewed. Twice and three times rotation of Euler angle already conducted and produced a matrix transformation different from the Kim-Akagi's model. Method of Fourier integral in the Voltage Source PWM Converter be done so a harmonics characteristic can be known and may be reduced. Value of Euler angles in frequency domain determined as a carrier harmonic waveforms. This approach is gives slightly different than that has understood so far.
\end{abstract}

Keywords - Power transformation, Euler angles rotation method, Voltage Source Converter, Harmonics reduced.

\section{INTRODUCTION}

$\mathrm{T}$ he active-reactive power theory known as pq-theory was first published in international journals in 1984 [1] and then refined into pqr-theory in advanced research conducted by Kim-Akagi [2] and other researchers [3,4]. This theory was developed to solve of harmonics problems caused by non-linear loads. Akagi used the term of power-filtering for this theory because its discussion includes about the active power filter [5]. At first, definition of instantaneous active power (p) and instantaneous reactive power (q) are called real power and imaginary power because there are no limits so charged on voltage and current. pq-theory in time domain still have conceptual limitations this theory is only completed for system 3 phase 3 wire without neutral component is a zero sequence voltage and/or a zero sequence current. Clarke transformation is used to change system of 3-phase 3-wire from abc-coordinates to $\alpha \beta$-coordinates. Meanwhile pqr-theory as improvements pq-theory applies to the 3-phase 4-wire in which presence of a neutral component is considered. Due to presence of a neutral component then analysis of activereactive power carried in pqr-coordinate. Circuit series consist of IGBT 3-phase 4-wire used in shunt active power filter with currents compensation fill in a closed loop systems [5].

The existence $r$-axis as the harmonics indicator related to the neutral component of the circuit 3-phase 4-wire PWM converter. If source is connected to non-linear load then current in the neutral wire is always exist, and if source is connected to linear load then current is zero at the neutral wire. Neutral components in the form of a zero sequence voltage and/or a zero sequence current on the neutral wire does not only affect instantaneous active power (p) but also instantaneous reactive power (q). The presence of neutral components can be observed in the oscillation phenomena that occur in the instantaneous active-reactive power when a zero sequence voltage and/or a zero sequence current was considered. As harmonics indicator, a system where if source is connected to non-linear load then neutral component will have a currents with certain value, it can not be eliminated completely though instantaneous reactive

${ }^{1}$ Indriarto Yuniantoro, Rudy Setiabudy, Ridwan Gunawan are with Departement of Electrical Engineering, Faculty of Engineering, Universitas Indonesia, Depok, 16242, Indonesia. E-mail: indy_singnet@yahoo.com; rudy@eng.ui.ac.id; ridwan@eng.ui.ac.id. power is compensated to zero. Compensated of a zero sequence voltage or a zero sequence current without energy storage system can be done in instantaneous reactive power but it is happens in the energy balance in the harmonic filter. Others way is change the balance of voltage or current in a rotating reference frame either to the method of control voltage or current control [6]. In experiments of the injection current compensation to non-linear loads, Akagi used a closed loop system that controls to currents in the active harmonic filter circuit to change the balance of instantaneous voltage or instantaneous current within pqr-coordinate framework.

Modify pq-theory Akagi in this associated with it. Developed pqr-coordinate in the mapping matrices model of Kim-Akagi [3] to explain the existence of the $r$ axis is related instantaneous active-reactive power and source of voltage/current in orthogonal vectors in tetrahedron space [7]. In perform of transformation so produce pqr-coordinate, Kim-Akagi doing two transformations in succession objective that projection voltage vector in $\mathrm{r}$-axis and $\mathrm{q}$-axis is zero [8]. Instantaneous active power (p) is result from scalar product between voltage-current and cause the flow of energy between two sub-systems. While instantaneous reactive power $(\mathrm{q})$ is result from vectors product between voltage-current and does not cause the flow of energy.

In this paper will be discussed of harmonic reduction by way coordinate transformation. A mathematical model is created as space vector transformation based on the Euler angle rotation method in which pqr-coordinates refers to mapping matrices model of Kim-Akagi. Matrix where transformation is obtained by twice and three time Euler angles rotation. Experiment with shunt active power filter not only on 3-phase 3-wire system but also on the 3-phase 4-wire system, and experiments used in a combination of asynchronous generator is reviewed. Matrix coordinate transformation used for harmonics reduction done especially at high frequencies, the way is controlled by active-reactive power (pq) on the PWM converter circuits. Control analysis of the active-reactive power in a pqr-coordinate is produced better than dqcoordinates

\section{HARMONICS REDUCED BY PWM CONVERTER}

\section{A. Harmonic reducing}

In electric power system a linear load is resulting in the current value proportional to the voltage and causing the current waveform same as with the voltage waveform, 
while non-linear loads cause different current waveforms from voltage and have been distorted. Non-linear loads in industrial and household electronic equipment are generally caused by semiconductor component are producers harmonic interference. Harmonics as a superposition waves at the fundamental and multiples thereof frequency affecting the electric power system and cause distortion in changes the pure sinusoidal waveform

Harmonic that cause defects on fundamental waves can cause interference and affect can in the form of increased resonance electrical distribution system, reduced the efficiency of power generation and transmission, reduced the insulation life of electric power distribution components, and interference protection systems, consumer appliances, and communication channels.

Reduced harmonics by way improved used in power electronics circuits is commonly referred to as active and passive harmonic filters. This filter has ability to filtering and reduction. Akagi particular develop an active harmonic filter is caused availability IGBT easily obtained in the market. Besides, active harmonic filter is cheaper, better equipment performance and size is also smaller than the passive harmonic filters.

Active harmonic filter consists of 2 kinds are pure and hybrid. Pure active harmonic filter is used as a voltage source series of PWM converter is equipped with capacitor, it can serves as a current source series of PWM converter is equipped with inductor. Hybrid filter composed of one or more voltage source PWM converter that connected to the passive filter components such as LC-circuit. This type of hybrids filter is more attractive than pure active filter primarily used in reducing harmonics in high frequency.

Compensation current control in space vector modulation on an active harmonic filter can be done by PWM current control used to adjust output compensation of current and voltage can also be done by using DSP (digital signal processing) for analysis in the frequency domain.

The output of the average value of the voltage or current in the modulation is determined by the period of the duty cycle of the square wave as a result of intersection between the source wave and sawtooth wave on the triangular carrier wave at PWM converter process. The square wave as harmonic reduction can be analyzed with the Fourier series. The objective is to increase the amount of output from a given switching frequency, and suitable strategy modulation topology developed by voltage source or current source PWM converter. Modulation process in the PWM converter is an intersection of reference wave with a sawtooth wave or triangular carrier wave [9].

Modulation is aims to create a pulsewidth which has the same value between the average voltage, Vdc, compared the source. The problem that arises is pulsewidth also contain undesirable harmonic components and must be eliminated too, therefore PWM converter should be controlled with switching ON-OFF to match the voltage or current desired. Switching process is performed as a harmonic elimination by PWM converter in closed-loop system to set the switching frequency of the phase leg condition.
Concept of clocking-pulse on PWM converter enables the carrier wave with half interval to eliminate low order of harmonics, where the frequency switching is used to keeping in shape the wave always to symmetry. In the picture-1 there are 2 square wave with cycle opposite Awave which started in the positive half cycle $(+\mathrm{Vdc})$ and B-wave started on the negative half cycle $(-\mathrm{Vdc})$. The square wave which is the intersection between the reference with a sawtooth and triangular carrier wave is the wave of PWM converter [9]. Respectively, has an angles period $00-3600$ to consist of $(2 \mathrm{~N}+1)$ square wave. The square wave produce harmonics at $\mathrm{h}=$ $1,3,5,7$. If $\alpha 1, \alpha 2, . . \alpha 2 \mathrm{~N}$ is a series that shows angle position of $(2 \mathrm{~N}+1)$ point of the Fourier series then it can be written for harmonic elimination as an equation follows:

$f(\omega t)=\sum_{n=1}^{\infty} a_{n} \sin n \omega t+b_{n} \cos n \omega t$

Where for a half-wave symmetry an equation is obtained as follows,

$a_{n}=\frac{2}{\pi} \int_{0}^{\pi} f(\omega t) \sin n \omega t d(\omega t)$

$b_{n}=\frac{2}{\pi} \int_{0}^{\pi} f(\omega t) \cos n \omega t d(\omega t)$

If equation-2 inserted into equation-1 then for the Awave obtained an equation as follow,

$a_{n}=\frac{2}{\pi} V_{d c}\left[\sum_{k=0}^{2 N-1}(-1)^{k+1} \int_{\alpha_{k}}^{\alpha_{k+1}} \sin n \omega t d(\omega t)+\right.$

$\left.\int_{\alpha_{2 N}}^{\pi} \sin n \omega t d(\omega t)\right]$

For $\alpha_{0}=0$. Whereas for B-wave obtained an equation as follow,

$a_{n}=( \pm 1) \frac{4}{n \pi} V_{d c}\left[1+\sum_{k=0}^{2 n}(-1)^{k}\left[\cos \left(n \alpha_{k}\right)\right]\right]$

In the same way, if equation- 3 inserted to equation-1 $\mathrm{b}_{\mathrm{n}}$-coefficients can be obtained as follows,

$b_{n}=( \pm 1) \frac{4}{n \pi} V_{d c}\left[\sum_{k=0}^{2 n}(-1)^{k}\left[\sin n \alpha_{k}\right]\right]$

\section{B. Review of pq and pqr-theory}

$\mathrm{Pq}$ and pqr theory was developed as a harmonic compensator on non-linear load conditions without energy storage components [5]. Pqr-theory is the development of pq-theory in the shunt active power filter for 3-phase 4-wire system. In pqr-theory analysis of instantaneous active-reactive power, especially for compensation neutral components must use the p-axis, qaxis and r-axis. Pq-theory or instantaneous activereactive power theory that proposed by Akagi, Kanazawa, Nabae different from previous theories because using Clarke transformation to explain real power, $\mathrm{p}$, and imaginary power, $\mathrm{q}$, in the 3-phase 3-wire system, while for the 3-phase 4-wire system review of the zero sequence component is influenced by the present of neutral component, $\mathrm{p} 0$.

$\mathrm{Pq}$ theory or instantaneous active-reactive power theory that developed by Akagi is obtained by multiplying the voltage and currents vector, and it produce power in the form of a matrix vector as follows, $\boldsymbol{S}=\overline{\boldsymbol{v}} \cdot \overline{\boldsymbol{\imath}}^{*}=\left(v_{\alpha}+j v_{\beta}\right)\left(i_{\alpha}-j i_{\beta}\right)=p+j q$

In matrix form, the real power and imaginary power in the pq-theory is given below,

$\left[\begin{array}{l}p \\ q\end{array}\right]=\left[\begin{array}{cc}i_{\alpha} & i_{\beta} \\ -i_{\beta} & i_{\alpha}\end{array}\right]\left[\begin{array}{l}v_{\alpha} \\ v_{\beta}\end{array}\right]$

In Park's transformation, dq-coordinate (rotating reference frame), real power and power relations are given imaginary d-axis is instantaneous active power (p) 
and $\mathrm{q}$-axis is the instantaneous reactive power (q). Equation-8 More can be written as follows,

$\left[\begin{array}{l}v_{d} \\ v_{q}\end{array}\right]=\left[\begin{array}{cc}\cos \omega t & \sin \omega t \\ -\sin \omega t & \cos \omega t\end{array}\right]\left[\begin{array}{l}v_{\alpha} \\ v_{\beta}\end{array}\right]=$

$\left[\begin{array}{cc}i_{\alpha}^{\prime} & i_{\beta}^{\prime} \\ -i_{\beta}^{\prime} & i_{\alpha}^{\prime}\end{array}\right]\left[\begin{array}{l}v_{\alpha} \\ v_{\beta}\end{array}\right]=\left[\begin{array}{l}p \\ q\end{array}\right]$

Physically as shown in Figure-2 below, the real power (p) as a scalar product is produces a certain value and cause the flow of energy, while the imaginary power (q) as a vector product is produces a certain value and direction but does not cause the flow of energy due to the imaginary rotating circular of the wire [10,11].

Pq-theory Akagi, though valid for the conditions of the current or voltage in sinusoidal and non-sinusoidal waveform has the disadvantage that is only valid for the 3-phase 3-wire system (without the zero sequence voltage or current). Required flow decomposition into orthogonal components of the voltage vector to produce a vector currents at particular coordinates (coordinate$\alpha \beta)$.

Definition of instantaneous active power in 3-phase circuit is given by the equation $\left|\mathrm{p}^{-}\right|=\mathrm{v}^{-} .^{-}$where "." indicates the dot product or scalar multiplication, instantaneous reactive power 3-phase circuit is given by $\mathrm{q}^{-}=\mathrm{v}^{-} \mathrm{x} \mathrm{i}^{-}$where " $\mathrm{x}$ " indicates the cross product or vector multiplication. According to Peng, properties the harmonic and compensation instantaneous activereactive power are derived from the basic components of positive sequence, $\mathrm{V}+$ or $\mathrm{I}+$ is symmetrical about the load current, while the negative sequence components derived from basic, V- or I- is asymmetric of the load current.

So far, authors are already understood many phenomena of electric power system, especially in the analysis of the induction generator using a $\alpha \beta$-coordinate and/or dqcoordinate. To solve the problem of harmonic and nonlinear loads then Kim-Akagi based on study of the tetrahedron space by Watanabe [7] proposes new coordinate transformation as refinement $\alpha \beta$-coordinate where the presence of a neutral component considered.

Developed pqr-coordinates on the mapping matrices model of Kim Akagi [8] is explain presence of r-axis component related neutral in instantaneous activereactive power with the voltage or current source orthogonal vectors which is in tetrahedron space. If source is connected to non-linear load then the neutral wire current is always there, if source is connected with linear load then the neutral wire current is zero.

Kim-Akagi's equation of the pqr-coordinate mapping matrices model is as follows,

$\left[\begin{array}{l}i_{p} \\ i_{q} \\ i_{r}\end{array}\right]=\left[\begin{array}{ccc}\frac{e_{\alpha}}{e_{\alpha \beta 0}} & \frac{e_{\beta}}{e_{\alpha \beta 0}} & \frac{e_{0}}{e_{\alpha \beta 0}} \\ \frac{-e_{\beta}}{e_{\alpha \beta}} & \frac{e_{\alpha}}{e_{\alpha \beta}} & 0 \\ \frac{-e_{0} e_{\alpha}}{e_{\alpha \beta} e_{\alpha \beta 0}} & \frac{-e_{\beta} e_{0}}{e_{\alpha \beta} e_{\alpha \beta 0}} & \frac{e_{\alpha \beta}}{e_{\alpha \beta 0}}\end{array}\right]$

\section{The Euler angle rotation method [12]}

Most of the instantaneous active-reactive theory (pq) above has been is developed in time domain. The model developed by Kim-Akagi constrain on the time domain. By Fourier transformation can be obtained instantaneous active-reactive power theory (pq) in the frequency domain for a non sinusoidal and non-linear loads such as in the following description.

Kim-Akagi's model as transformation a rotation of twice Euler angle is developed into three time Euler angles that it seems changes. By giving certain constraints of apply to transformation of three angle, and the interim is resulted of the simulation Matlab which have shown the presence of r-axis has a significant compare as shown Figure-3 below, it can be concluded that analysis the Euler angle rotation method generated for the better. Multiplication Euler angles for 2 variable angles $\theta 1, \theta 2$ are as follows:

$\mathrm{R}\left(\theta_{1}, \theta_{2}\right)=\mathrm{R}_{\mathrm{N}}\left(\theta_{2}\right) \mathrm{R}_{\mathrm{z}}\left(\theta_{1}\right)$,

$$
\begin{aligned}
& =\left[\begin{array}{ccc}
\cos \theta_{2} & 0 & \sin \theta_{2} \\
0 & 1 & 0 \\
-\sin \theta_{2} & 0 & \cos \theta_{2}
\end{array}\right]\left[\begin{array}{ccc}
\cos \theta_{1} & \sin \theta_{1} & 0 \\
-\sin \theta_{1} & \cos \theta_{1} & 0 \\
0 & 0 & 1
\end{array}\right] \\
& =\left[\begin{array}{ccc}
\cos \theta_{2} \cos \theta_{1} & \cos \theta_{2} \sin \theta_{1} & \sin \theta_{2} \\
-\sin \theta_{1} & \cos \theta_{1} & 0 \\
-\sin \theta_{2} \cos \theta_{1} & -\sin \theta_{2} \sin \theta_{1} & \cos \theta_{2}
\end{array}\right]
\end{aligned}
$$

in this case $R_{N}\left(\theta_{2}\right)=R_{y}\left(\theta_{2}\right)$ is rotation on normal direction in the y-axis. Equation-11 is the same result with Kim-Akagi's mapping matrices model are as in equation-10 above. Multiplication Euler angles for 3 variable angles $\theta_{1}, \theta_{2}, \theta_{3}$ are as follows,

$\mathrm{R}\left(\theta_{1}, \theta_{2}, \theta_{3}\right)=\mathbf{R}_{\mathrm{z}}\left(\theta_{3}\right) \mathbf{R}_{\mathrm{N}}\left(\theta_{2}\right) \mathbf{R}_{\mathrm{z}}\left(\theta_{1}\right)$

$$
\begin{aligned}
= & {\left[\begin{array}{ccc}
\cos \theta_{3} & -\sin \theta_{3} & 0 \\
\sin \theta_{3} & \cos \theta_{3} & 0 \\
0 & 0 & 1
\end{array}\right] \mathrm{x} } \\
& {\left[\begin{array}{ccc}
\cos \theta_{2} \cos \theta_{1} & -\cos \theta_{2} \sin \theta_{1} & -\sin \theta_{2} \\
\sin \theta_{1} & \cos \theta_{1} & 0 \\
\sin \theta_{2} \cos \theta_{1} & -\sin \theta_{2} \sin \theta_{1} & \cos \theta_{2}
\end{array}\right] }
\end{aligned}
$$

By inserting constraint of each axis to the validity of the Euler angles, then the result of the matrix multiplication in equation-12 and,

For $\alpha$-axis $\rightarrow$ p-axis:

$\mathrm{f}\left(\theta_{1}, \theta_{2}, \theta_{3}\right)$ as a fuction from $\theta_{1}, \theta_{2}, \theta_{3}$

For $\beta$-axis $\rightarrow$ q-axis:

$\mathrm{f}\left(\theta_{1}, \theta_{3}\right)$ as a fuction from $\theta_{1}, \theta_{3}$, where $\theta_{2}=0$

For -axis0 $\rightarrow$ r-axis:

$\mathrm{f}\left(\theta_{2}\right)$ as a fuction from $\theta_{2}$, where $\theta_{1}, \theta_{3}=0$

So the analytic geometry of the rotation matrix can be written as follow;

$\mathrm{R}\left(\theta_{1}, \theta_{2}, \theta_{3}\right)=$

$\left[\begin{array}{ccc}\cos \theta_{2} \cos \left(\theta_{3}+\theta_{1}\right) & \sin \left(\theta_{3}+\theta_{1}\right) & \sin \theta_{2} \\ -\sin \left(\theta_{3}+\theta_{1}\right) & \cos \left(\theta_{3}+\theta_{1}\right) & 0 \\ -\sin \theta_{2} & 0 & \cos \theta_{2}\end{array}\right]$

So rotation for 3 variable Euler angles is obtained a transformation matrix as follow;

$\left[\begin{array}{l}X_{p} \\ X_{q} \\ X_{r}\end{array}\right]=\sqrt{\frac{2}{3}}\left[\begin{array}{ccc}\cos \theta_{2} \cos \left(\theta_{3}+\theta_{1}\right) & \sin \left(\theta_{3}+\theta_{1}\right) & \sin \theta_{2} \\ -\sin \left(\theta_{3}+\theta_{1}\right) & \cos \left(\theta_{3}+\theta_{1}\right) & 0 \\ -\sin \theta_{2} & 0 & \cos \theta_{2}\end{array}\right]$

$\left[\begin{array}{ccc}1 & -\frac{1}{2} & -\frac{1}{2} \\ 0 & \frac{\sqrt{3}}{2} & -\frac{\sqrt{3}}{2} \\ \frac{1}{\sqrt{2}} & \frac{1}{\sqrt{2}} & \frac{1}{\sqrt{2}}\end{array}\right]\left[\begin{array}{l}X_{a} \\ X_{b} \\ X_{c}\end{array}\right]$

in this case $X \equiv i, V$ (is current or voltage).

\section{METHOD AND EXPERIMENT}

\section{A. Configuration of shunt active filter for pq-Theory and pqr-Theory}

Study of some combination of active power filter has been done by Aredes [13.14], with scheme circuits such as Figure-4,5 on the side, and the results obtained 
indicate the presence of instantaneous active-reactive power effect not only on 3-phase 4-wire system but also on 3 phase 3 wire system as in Akagi's pq-theory. Conducted by Aredes et al actually have also been done by Akagi using Voltage-Source Inverter (VSI) schemes is produce only limited conclusions on 3-phase 3-wire system.

\section{B. Asynchronous generators plus active power filter without current transformer}

Asynchronous generators with $\mathrm{Y}$-connection associated with a series circuit the current transformer and into the space vector modulation circuit for pqr-coordinate transformation. Asynchronous generators were derived from the DC drive motor with variable speed-controlled cycle. Flow meters (ammeters) mounted on the load side and then made observations.

\section{Hybrid active harmonic filter}

Active harmonic filter consists of a combination of hybrid active filter, passive filter, and a three-phase transformer. Hybrid filter can be used as a filter as well as the harmonic passive filter as a generally. Active filter tasks not only to compensate for harmonic currents produced but also to achieve. "harmonic isolation" between source and load. Consequently, there is no harmonic resonance occurs and no harmonic current that goes to the source because it can cause damage.

Usually used a diode rectifier circuit is itself only the problem of harmonic generating sources that are used for IGBT active filter. Circuit with a voltage source PWM converter system used IGBT has been widely used in the industry, household appliances and transport.

\section{RESULT AND DISCUSSION}

Matrix pqr-coordinate transformation of Kim-Akagi can be reformulated with rotation of twice Euler angles method, and it can even be further expanded with three time Euler angles as a correction factor. Matrix coordinates transformation used for harmonics reduction done especially at high frequencies, the way to control the active-reactive power (pq) on the PWM converter circuits.

For 3-phase 3-wire system, active harmonic filter is used as a hybrid combination of IGBT switching circuits and passive filter capacitor, inductor and/or resistors. While for system 3-phase 4-wire system, this study is being made and not yet completed. In 3-phase 3-wire system is used to prove instantaneous active-reactive theory pq-theory while the system 3-phase 4-wire theory proves particularly pqr-coordinate transformation.

\section{ACKNOWLEDGEMENT}

This work was supported by Decentralization Research Grant allocation for University of Trisakti Jakarta under contract no. 002/K3/KM/SPK/2013.

\section{REFERENCES}

[1] H.Akagi, Y.Kanazawa and A.Nabae, Instantaneous Reactive Power Compensators Comprising Switching Devices without Energy Storage Components. IEEE Transaction on Industry Application, vol. IA-20, No. 3, pp. 625-630. May/June 1984.

2] H.Kim and H.Akagi. The Instantaneous Power Theory on the Rotating p-q-r Reference Frames. IEEE International Conference on Power Electronics and Drive System (PEDS), pp 422-427. July 1999. Hong Kong.

[3] M.Aredes, J.Haffner, K.Heumann. Three-Phasa Four-Wire Shunt Active Filter Control Strategies. IEEE Transaction on Power Electronics, vol. 12, No.2, pp. 311-318, March 1997.

[4] E.H.Watanabe, H.Akagi and M.Aredes, Instantaneous p-q Power Theory for Compensating Nonsinusoidal Systems. International School on Nonsinusoidal Currents and Compensation. Lagow, Poland, 2008.

[5] H.Akagi, E.H.Watanabe and M,Aredes. Instantaneous Power Theory and Applications to Power Conditioning. J. Wiley-IEEE Press, 2007.

[6] M.Aredes, H. Akagi, E.H. Watanabe, E. Vergara and L.F. Encarnação. Comparisons Between the p--q and p--q--r Theories in Three-Phase Four-Wire Systems. IEEE Transaction on Power Electronics. vol. 24. no. 4. pp 924-933. April 2009.

[7] E.H.Watanabe, R.M.Stephan and M.Aredes. New Concepts of Instantaneous Active and Reactive Power in Electrical Systems with Generic Loads. IEEE Transaction on Power Delivery, vol.8, No.2, pp.697-703. April 1993.

[8] H.Kim and H.Akagi. The Instantaneous Power Theory Based on Mapping Matrices in Three-phase Four-wire Systems. PCC Nagaoka 1997

[9] D.G. Holmes, T.A. Lipo. Pulse Width Modulation for Power Converter. J.Wiley-IEEE Press, 2003.

[10] F.Z. Peng and J.S. Lai. Generalized Instantaneous Reactive Power Theory for Three-phase Power Systems. IEEE Transaction on Instrumentation and Measurement, vol. 45, No. 1, pp. 293-297. February 1996.

[11] F.Z.Peng, G.W.Ott and D.J. Adam. Harmonic and Reactive Power Componen Based on the Generalized Instantaneous Reactive Power Theory for 3-phase 4-wire Systems.

[12] I.Yuniantoro, R.Gunawan and R.Setiabudy. The pqr-coordinate in the Mapping Matrices Model of Kim-Akagi on Power Transformation based on Euler Angle Rotation Method. The 13th International Conference on Quality in Research (QiR) 2013, FTUI, pp. 1022-1027. Jogjakarta, June 25-28, 2013.

[13] M.Aredes, H. Akagi, E.H. Watanabe, E. Vergara and L.F. Encarnação. Comparisons Between the p--q and p--q--r Theories in Three-Phase Four-Wire Systems. IEEE Transaction on Power Electronics. vol. 24. no. 4. pp 924-933. April 2009.

[14] M.Aredes and E.H. Watanabe. New Control Algorithms for Series and Shunt Three-Phase Four-Wire Active Power Filters. IEEE Transaction on Power Delivery, Vol. 10, No. 3, pp. 1649. July 1995.
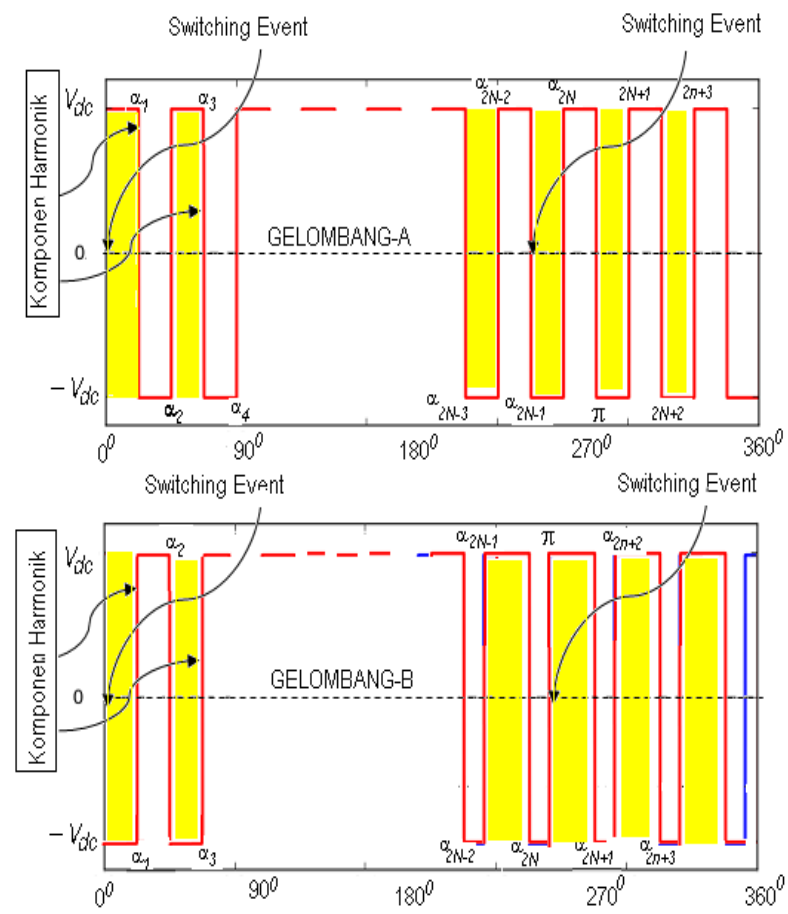

Figure 1. Harmonics in the square wave 

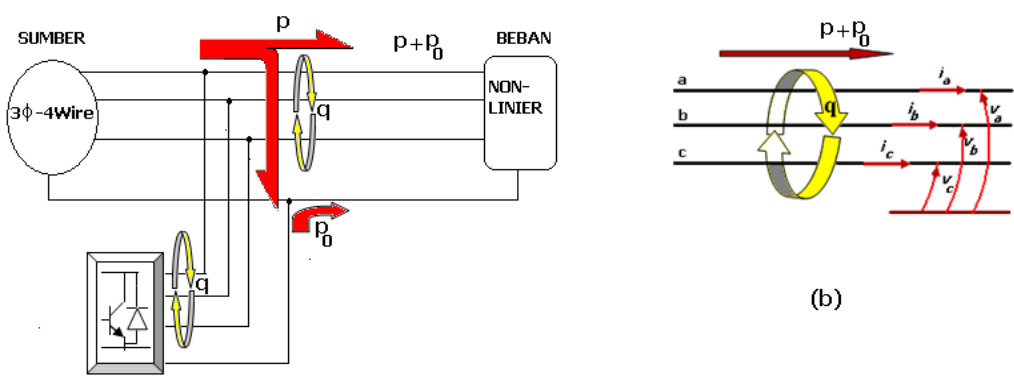

(a)

Figure 2. Peng's Illustration on instantaneous active power (p) and instantaneous reactive power (q)

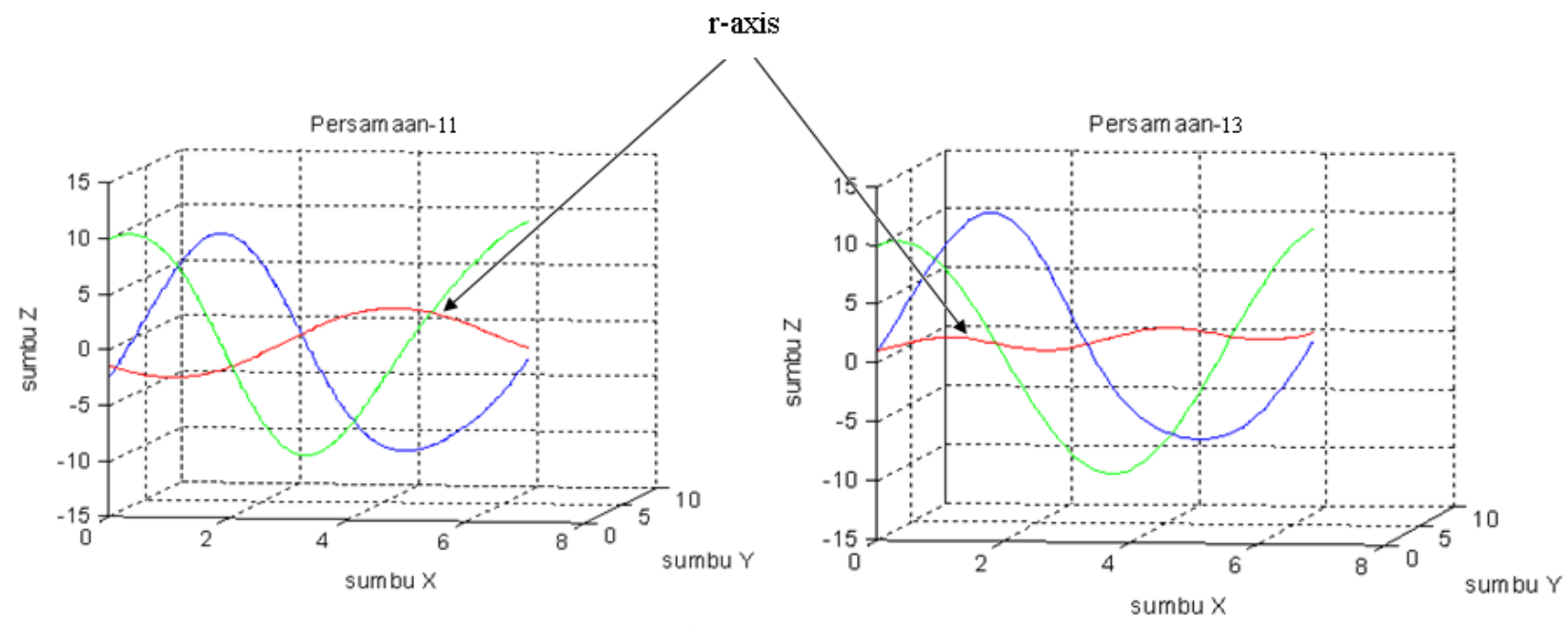

Figure 3. Comparison of the existence r-axes on the pqr-coordinate

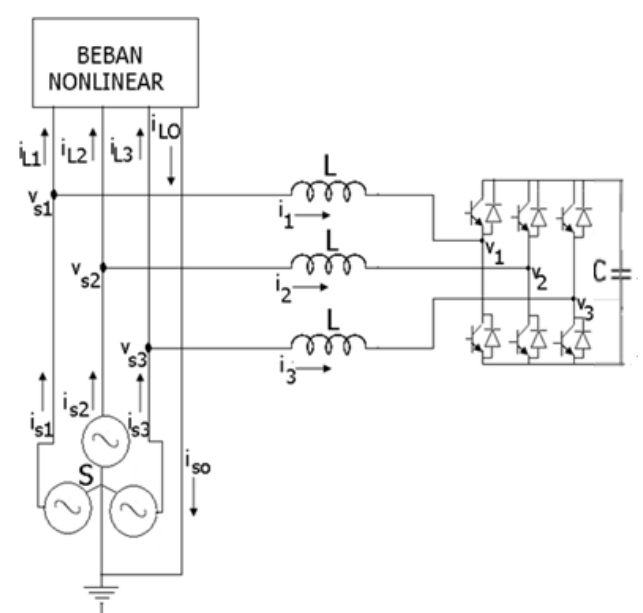

Figure 4. Schematic circuit of shunt active power filter 3-phase 3-wire

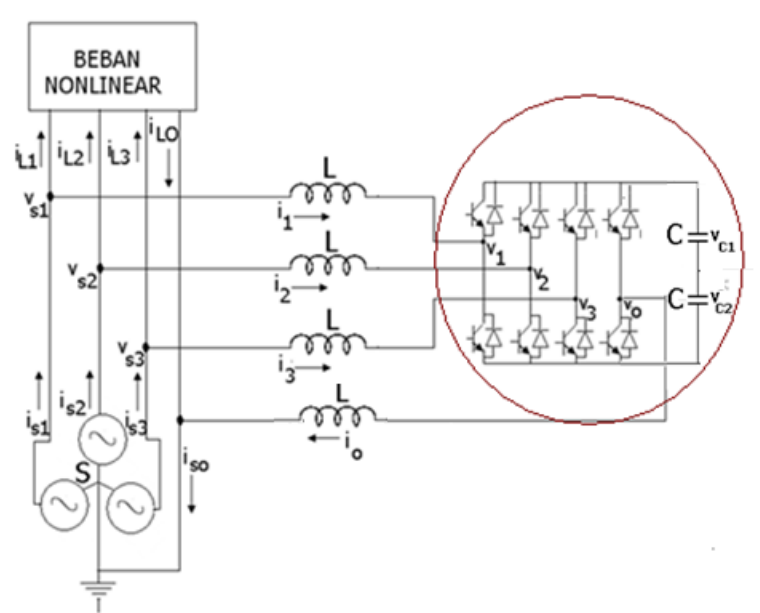

Figure 5. Schematic circuit of shunt active power filter 3-phase 4-wire 